\title{
Ultrasound-targeted microbubble destruction promotes myocardial angiogenesis and functional improvements in rat model of diabetic cardiomyopathy
}

\author{
Xijun Zhang ${ }^{1 \dagger}$, Xinqiao Tian ${ }^{1 \dagger}$, Peng $\mathrm{Li}^{2}$, Haohui Zhu ${ }^{1}$, Nanqian Zhou' ${ }^{1}$, Zhixin Fang ${ }^{2}$, Yuping Yang ${ }^{2}$, Yun Jing ${ }^{2}$ \\ and Jianjun Yuan ${ }^{1 *}$ (1)
}

\begin{abstract}
Background: Microvascular insufficiency plays an important role in the development of diabetic cardiomyopathy (DCM), therapeutic angiogenesis has been mainly used for the treatment of ischemic diseases. This study sought to verify the preclinical performance of SonoVue microbubbles (MB) combined ultrasound (US) treatment on myocardial angiogenesis in the rat model of DCM and investigate the optimal ultrasonic parameters.

Methods: The male Sprague-Dawley (SD) rats were induced DCM by streptozotocin through intraperitoneal injecting and fed with high-fat diet. After the DCM model was established, the rats were divided into the normal group, DCM model group, and US + MB group, while the US + MB group was divided into four subsets according to different pulse lengths (PL) (8 cycles; 18 cycle;26 cycle; 36 cycle). After all interventions, all rats underwent conventional echocardiography to examine the cardiac function. The rats were sacrificed and myocardial tissue was examined by histology and morphometry evaluations to detect the myocardial protective effect of SonoVue MBs using US techniques.

Results: From morphologic observation and echocardiography, the DCM rats had a series of structural abnormalities of cardiac myocardium compared to the normal rats. The US-MB groups exerted cardioprotective effect in DCM rats, improved reparative neovascularization and increased cardiac perfusion, while the 26 cycle group showed significant therapeutic effects on the cardiac functions in DCM rats.

Conclusion: This strategy using SonoVue MB and US can improve the efficacy of angiogenesis, even reverse the progress of cardiac dysfunction and pathological abnormalities, especially using the 26 cycle parameters. Under further study, this combined strategy might provide a novel approach for early intervention of DCM in diabetic patients.
\end{abstract}

Keywords: Diabetic cardiomyopathy, Ultrasound microbubble contrast, Angiogenesis, Animal model

*Correspondence: 342354073@qq.com

tXijun Zhang and Xinqiao Tian equally contribute to this work; they both are listed as the co-first authors

${ }^{1}$ Department of Ultrasonography, Zhengzhou University People's Hospital, Henan Provincial People's Hospital, Henan University People's Hospital, NO. 7 Weiwu Road, Zhengzhou 450003, Henan, China

Full list of author information is available at the end of the article

\section{Background}

Diabetic cardiomyopathy (DCM), caused by diabetes, is a common cardiovascular complication independent of coronary artery disease and hypertension [1]. It has a set of changes in the myocardial structure and function and has been receiving extensive attention due to the associated morbidity and fatality rates [2, 3]. However, DCM is often neglected in the clinical process because of no 
special symptoms in the early stage and lack of specialized treatment strategies [3-6]. Therefore, reveal the subtle changes of cardiac mechanics are important for patient management and intervention.

Microvascular insufficiency plays an important role in the development of DCM [4]. Accumulating evidence shows that the impaired angiogenic response to chronic ischemia may lead to reduced perfusion of myocardium, which finally contributes to interstitial fibrosis, oxidative tissue injury, and heart failure [7, 8]. Therefore, promoting cardiac angiogenesis and improving microcirculation function has become a potential therapeutic target for the treatment of DCM [8-11].

Recently, therapeutic angiogenesis has been mainly used for the treatment of ischemic diseases $[9,10]$. However, previous studies have reported the delivery approach of proteins and genes, including intracoronary, intrapericardial or myocardial administration, are inefficient and high risk that prevent its clinical applications. At the moment, ultrasound-targeted microbubble destruction (UTMD) has been developed as a noninvasive and target specific method in angiogenesis therapy of cardiovascular disease $[5,12,13]$.

UTMD technique has acquired much attention as an effective method to deliver basic fibroblast growth factor
(bFGF) to the heart, and the resulting growth factor therapy has obviously reverse cardiac dysfunction and even the structure of damaged cardiac tissues [12]. However, bFGF has a potential tumorigenic ability, so the lack of safety limits its application in vivo.

As an ultrasound contrast agent, SonoVue (Bracco International BV, Amsterdam, Netherlands) showed high safety, good contrast-enhanced images in clinical use [14-16]. The present study aims to investigate the effects of SonoVue/UTMD combined treatment on myocardial angiogenesis and inspect the optimal ultrasonic parameters in a rat model of DCM. The experimental details were shown in Fig. 1.

\section{Methods}

\section{Microbubble preparation}

We used an ultrasound contrast agent, Sonovue (Bracco International BV, Amsterdam, Netherlands) as microbubbles (MB). It was dissolved in $5 \mathrm{ml}$ physiological saline to make a SonoVue colloid dispersion, which contained $60.7 \mathrm{mg}$ SF6 and $25 \mathrm{mg}$ lyophilized powder. The MB concentration in the solution was measured by cell counting microscopic method. The MB concentration in the suspension was about $2 \times 10^{8}$ bubble $/ \mathrm{mL}$ with an average diameter of $3.4 \mu \mathrm{m}$. The diluted solution of microbubble

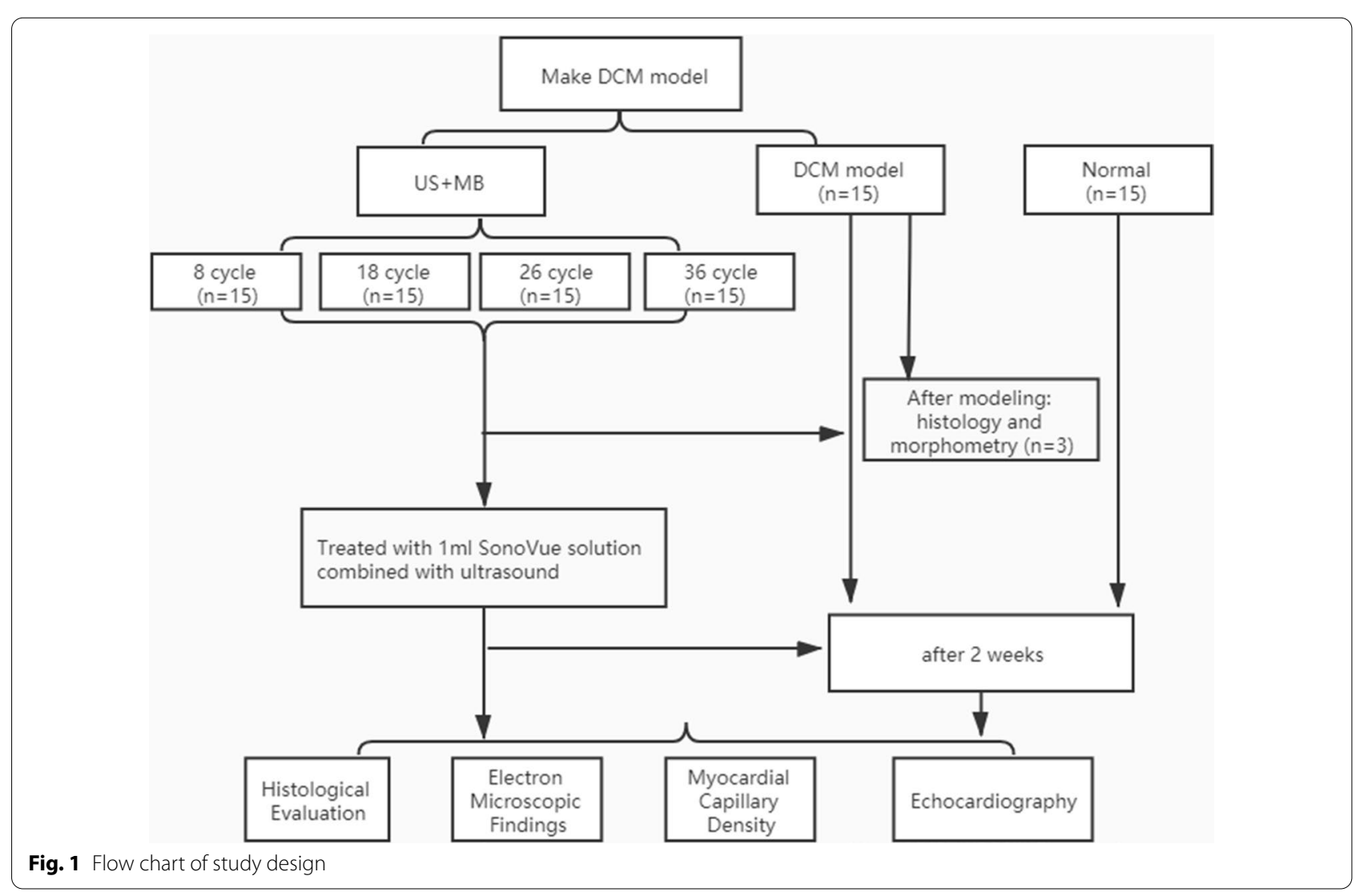


suspension $(1 \mathrm{~mL}$ microbubble suspension in $19 \mathrm{~mL}$ normal saline, which was diluted by 400 fold) was used in therapy. The therapeutic dose was about $1 \mathrm{~mL} /$ piece, and the continuous infusion lasted for $20 \mathrm{~min}$.

\section{DCM animal models}

All animal experiments in this study were approved by the Animal Care and Use Committee of Xinxiang Medical College and conducted by the "Guide for the Care and Use of Laboratory Animals" from the National Institutes of Health. Sprague- Dawley (SD) rats were widely used as an animal model for studying diabetes mellitus (DM), which were suitable for the evaluation of cardiac function $[5,6,12,13,17]$. Male rats (7-8 weeks, 160-180 g) were provided by the Experimental Animal Center of Zhengzhou University. Diabetes was induced by a single intraperitoneal injection of streptozotocin (STZ, Sigma Corporation, USA) at $65 \mathrm{mg} / \mathrm{kg}$ after $12 \mathrm{~h}$ of fasting, which had a similar progression of human's DM. The normal control rats were administered with the same volume of normal saline. After one week, the DM rats were provided with a fresh high fat-diet (basal diet added with $18 \% \mathrm{~W} / \mathrm{W}$ fat oil and $20 \% \mathrm{~W} / \mathrm{W}$ glucose) for 8 consecutive weeks, while the control group was fed with a normal diet. The fasting tail blood glucose was measured on the 3rd day, 7th day, and 9th week after the STZ injection. The rats with blood glucose over $16.6 \mathrm{mmol} / \mathrm{L}$ were selected as DM rats using in the following study [17-19].

\section{Groups and treatments of animals}

The rats were anesthetized using an intraperitoneal injection of $30 \mathrm{mg} / \mathrm{kg}$ sodium pentobarbital. The thoracic region was carefully shaved and placed supine on a warm procedure board. The VINNO 70 color Doppler diagnosis system (VINNO, Suzhou, China) combined with VFLASH (a software that can manipulate microbubble cavitation) was utilized to generate the UTMD effect, which was performed using a $14 \mathrm{MHz}$ linear-array transducer. The traditional echocardiographic measurements were obtained by M-mode echocardiography at left ventricular long-axis views (Fig. 2a), including the left ventricular internal dimension at end-diastole (LVIDd), the thickness of interventricular septum at end-diastolic (IVS), left ventricular fraction shortening (LVFS) and left ventricular ejection fraction (EF).

After satisfactory images were obtained (Fig. 2b), a dosage of $1 \mathrm{ml}$ diluted SonoVue solution was administered into the tail vein continuously over $20 \mathrm{~min}$ (Fig. 2c). At the same time, the VFLASH mode of the scanner was switched on for UTMD (frequency: $4 \mathrm{MHz}$, pulse frequency: $20 \mathrm{~Hz}$, exposure time $=1.2 \mathrm{~s}$, US exposure duration per time $=2 \mathrm{~s}$ ) and the heart was in the region of interest (ROI) during the experiment.

The animals were randomly divided into three groups: (1) Normal group $(n=15)$ : non-diabetic rats were injected $1 \mathrm{ml}$ saline solution; (2) DCM model group $(\mathrm{n}=15)$ : DCM rats were injected $1 \mathrm{ml}$ saline solution. (3) US + MB group: DCM rats were treated with $1 \mathrm{ml}$ SonoVue solution combined with ultrasound (US). The third group was divided into four subsets according to different pulse lengths (PL) (8 cycles; 18 cycle; 26 cycle; 36 cycle) ( $n=15$ for each group). For all animals, the second treatments were processed after one week of the first treatments.

\section{Histology and morphometry}

All specimens were anesthetized using an intraperitoneal injection of $30 \mathrm{mg} / \mathrm{kg}$ sodium pentobarbital, then euthanized by thoracotomy and heart removal. Three rats from the control group and DCM group were euthanized after modeling to evaluate the pathological changes of the pancreas and myocardium. After two weeks of all
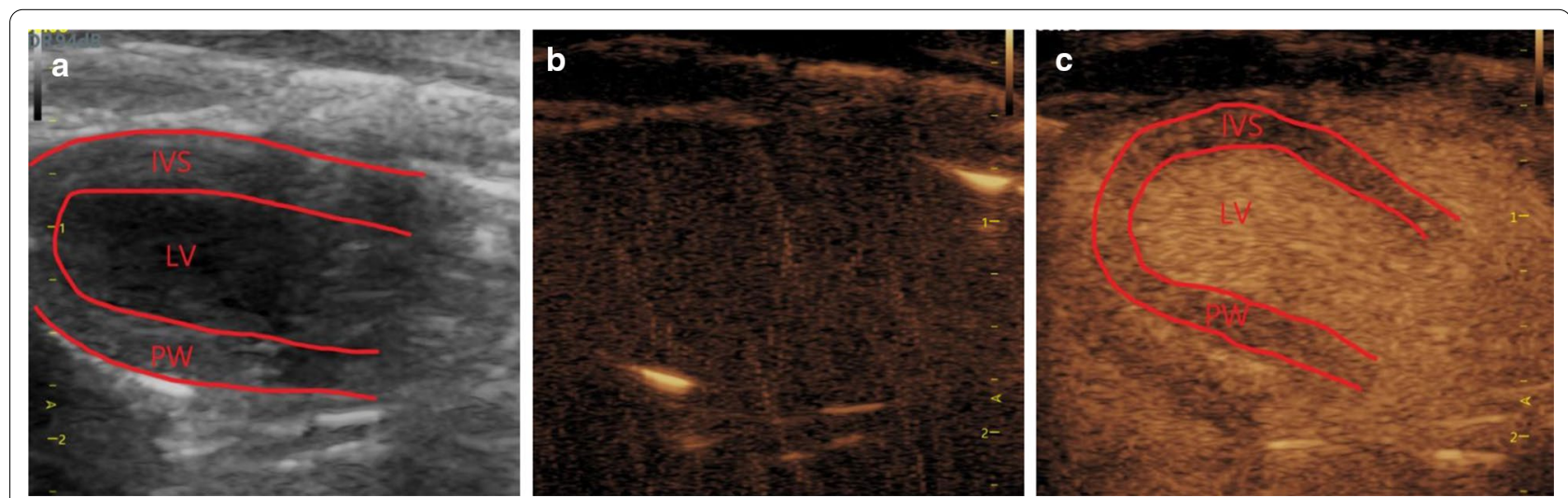

Fig. 2 Contrast ultrasonography of SonoVue MB in rat heart. a 2D echocardiograms, b ultrasound images before the filling of MB. $\mathbf{c}$ left ventricular was filled with $M B$ 
the intervention, the rats were sacrificed, then the left ventricular myocardium was fixed with $10 \%$ paraformaldehyde and embedded in paraffin. Sections of papillary muscle (about $5 \mathrm{~mm}$ thick) were stained with hematoxylin and eosin (HE) to observe the morphology.

CD31 immunohistochemistry was used to identify the myocardial capillary density (MCD). A total of 20 high power fields $(400 \times)$ were randomly selected and the number of brown-stained capillaries was counted and averaged as MCD [20].

\section{Statistical analysis}

All the data was graphed and analyzed in Microsoft Excel (Microsoft Corporation, Redmond, WA, USA) and SPSS statistical software (13.0 SPSS Inc., Chicago, IL, USA). The data were expressed as the mean \pm SD. Statistical comparisons were determined using one-way ANOVA and LSD-t test. Dunnett's T3 test was performed on the variance, and significance levels were defined at $P<0.05$.

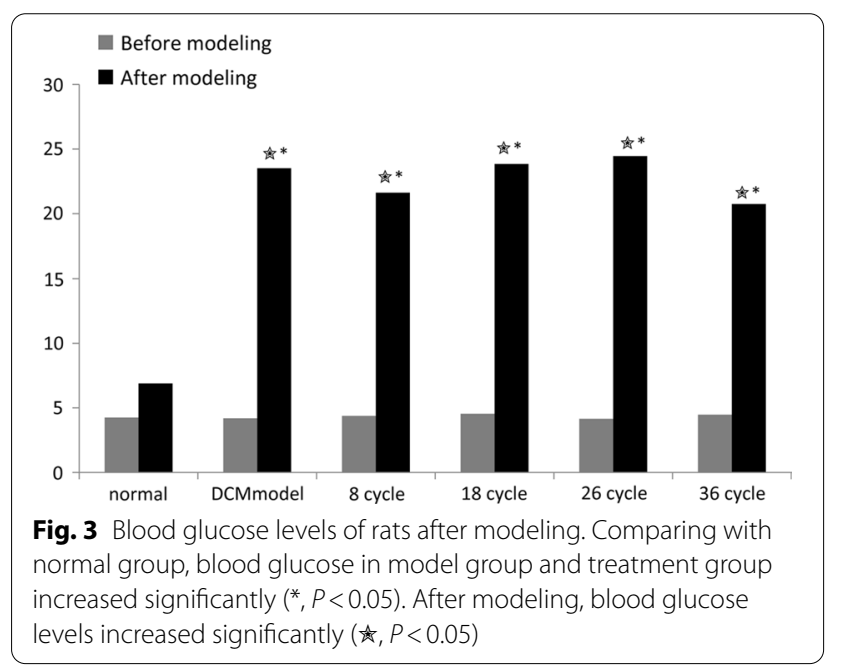

\section{Results}

General condition of experimental rats

After two weeks of all intervention, the final number of rats completed the study in each group were: 12 in the DCM model group, 12 in the 8 cycle group, 14 in the 18 cycle group, 13 in the 26 cycle group, 12 in the 36 cycle group, and 14 in the normal control group.

For blood glucose analysis, rats fasted overnight and blood samples were collected. Serum was separated and levels of glucose were measured. The glucose values after modeling were exceeding $16.6 \mathrm{mmol} / \mathrm{L}$. There was a significant increase in blood glucose levels after modeling compared to those before modeling with a $P$-value $<0.05$ (Fig. 3) for all groups except the normal control group. A distinct difference was observed in blood glucose levels between DCM rats and the normal control groups $(P<0.05$, Fig. 3).

\section{Histological evaluation}

After modeling, as shown in Fig. 4, the pancreatic tissue structure was complete and regular, the islet cells were compact and uniform, and the cytoplasm was abundant without vacuolation (Fig. 4a). Therefore, the pancreatic tissue of the DCM rats was seriously damaged, and the islet cells showed degenerative atrophy, only a few degenerative islet cells were sparsely distributed (Fig. 4b).

After two weeks of all interventions, as shown in Fig. 5, the cardiomyocytes were intact with a normal clear structure, and the nucleus was oval and located in the center of the normal group (Fig. 5a), while a large number of hypertrophic and necrotic cardiomyocytes were observed in the DCM model group, which were distributed in an altered and disorganized arrangement with obvious vacuolization (Fig. 5b). Nuclear pyknosis and myocardial fibrosis increased significantly in the DCM model group. The DCM rats had a set of structural
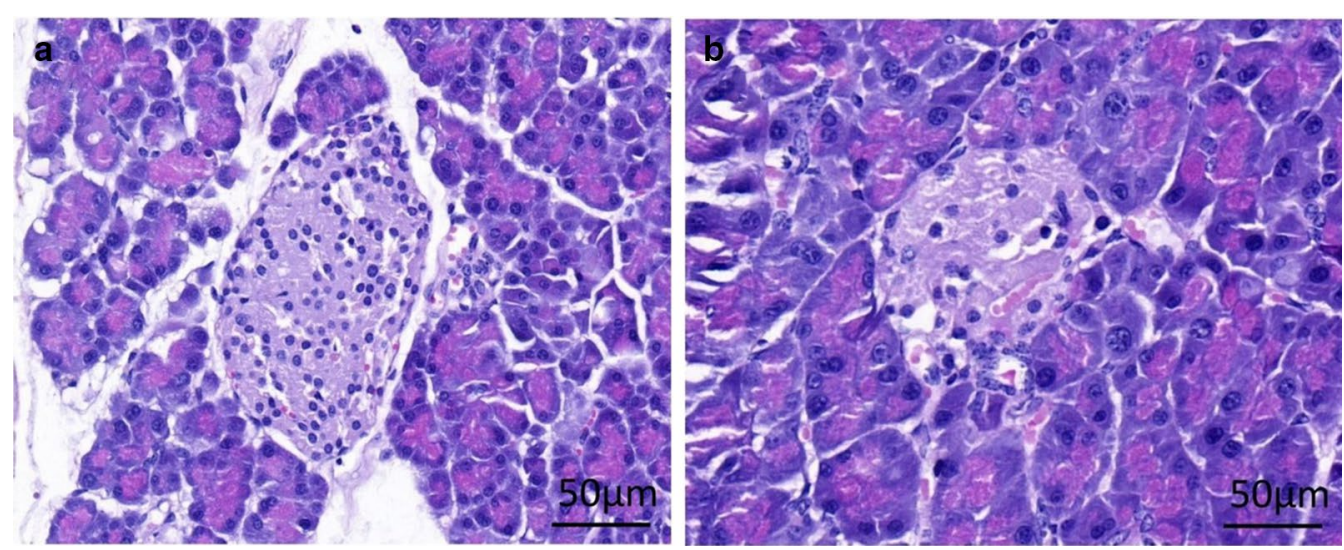

Fig. 4 The histological changes of the pancreas after modeling. a The normal group; $\mathbf{b}$ the DCM model group 

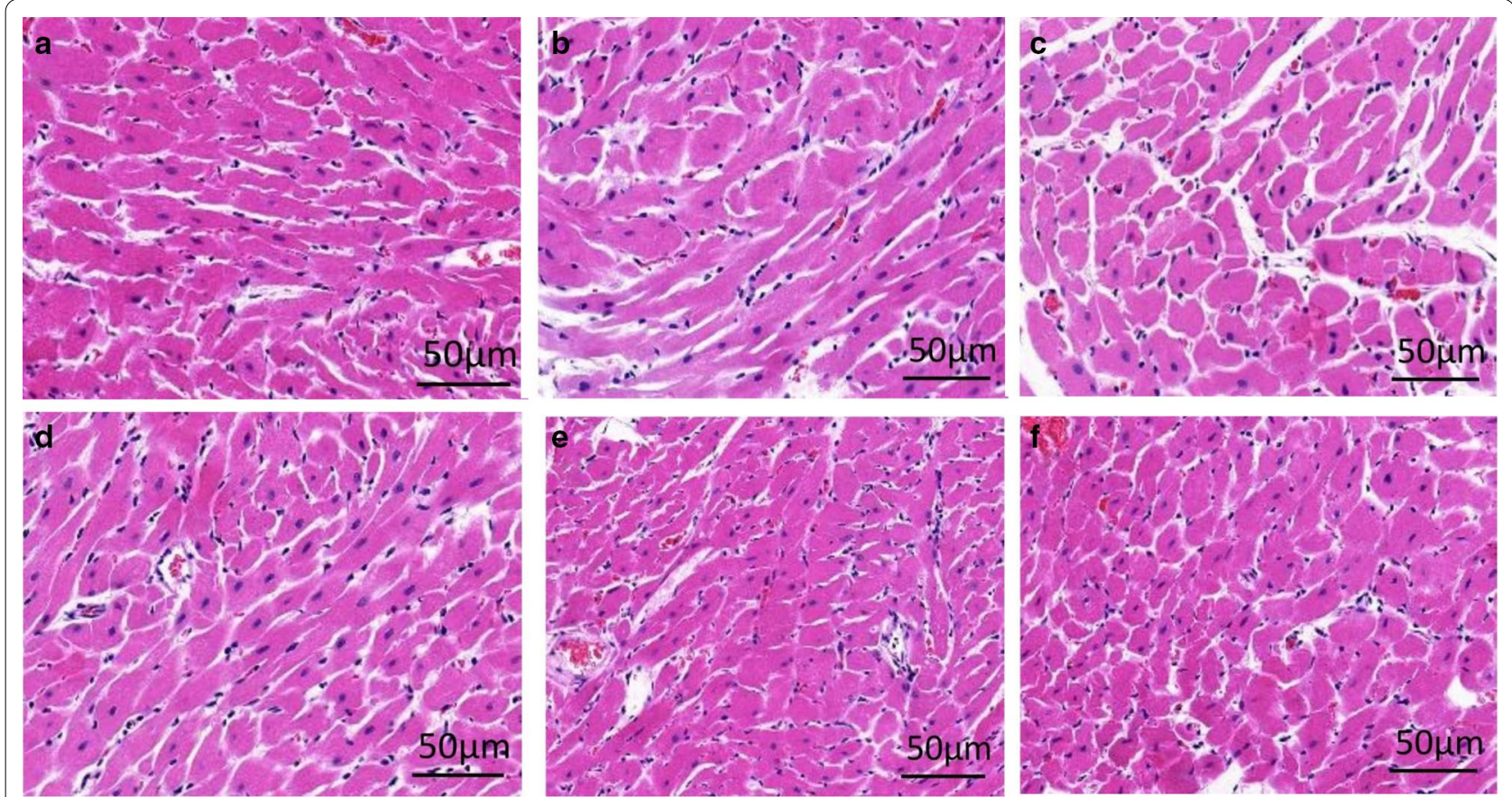

Fig. 5 The histological changes of the heart after intervention. a Normal group, b DCM model group, c 8 cycles group, d 18 cycle group, e 26 cycle group, $\mathbf{f} 36$ cycle group

abnormalities in the heart, and therefore, the US $+\mathrm{MB}$ treatment groups (Fig. $5 \mathrm{~d}-\mathrm{f}$ ) showed decreased hypertrophy, fibrosis and cardiomyocyte lesions.

\section{Electron microscopic findings}

To evaluate the changes of microvascular under different intervention conditions, the ultrastructural changes of the cardiac microvascular were examined under electron microscope. As shown in Fig. 6, in the normal group, the surface of cardiac microvascular was smooth, there was no obvious convexity, and the end structure of blood vessels was intact, while the surface of cardiac microvascular in the model control group was more burred and continuality was missing, the endothelial cell membrane was incomplete, and the heterochromatin of the inner nucleus increased and agglomerated, which indicated the pathological state of these microvessels. In the 36 cycle group, myocardial arterioles were not smooth, continuity was missing, endothelial cell membrane was incomplete, nuclear heterochromatin increased and agglomerated, showing a state of injury. In the 8 cycle group, 18 cycle group, and 26 cycle group, the surface of cardiac microvascular were smooth and continuous, the endothelial cell membrane was intact, and the cytoplasmic mitochondria were angiogenesis, while the myocardial samples in the 26 cycle group showed the relatively well-integrated ultrastructures compared with other US-MB treatment and DCM group.

\section{Echocardiography evaluation}

The traditional echocardiographic measurements including LVIDd, IVS, LVEF, LVFS and HR were acquired to estimate the improvement of global myocardial contractile function after two weeks of all intervention (Table 1). There was statistically significant difference in LVEF, LVFS and HR in the DCM model group and normal control group $(P<0.05)$. A distinct improvement was observed in DCM model rats in the levels of the LVEF, LVFS and HR with a $P$ value $<0.05$ after UTMD intervention. The LVEF and LVFS in the US + MB subgroup were significantly increased compared with the DCM model group (all $P<0.05$ ). Among all the US $+\mathrm{MB}$ groups, the 26 cycle group showed the highest LVEF and LVFS after treatment, and had no statistically significance when compared to the normal group $(P>0.05)$. There was no statistical significant difference in HR in the 8 cycle group and DCM group after the intervention, but a distinct difference was observed between the DCM group and other US-MB groups in HR with a $P$ value $<0.05$. There was a significant increase in the level of HR in the US-MB group but they still did not reach the levels of the normal control rats. There was no statistically significant 

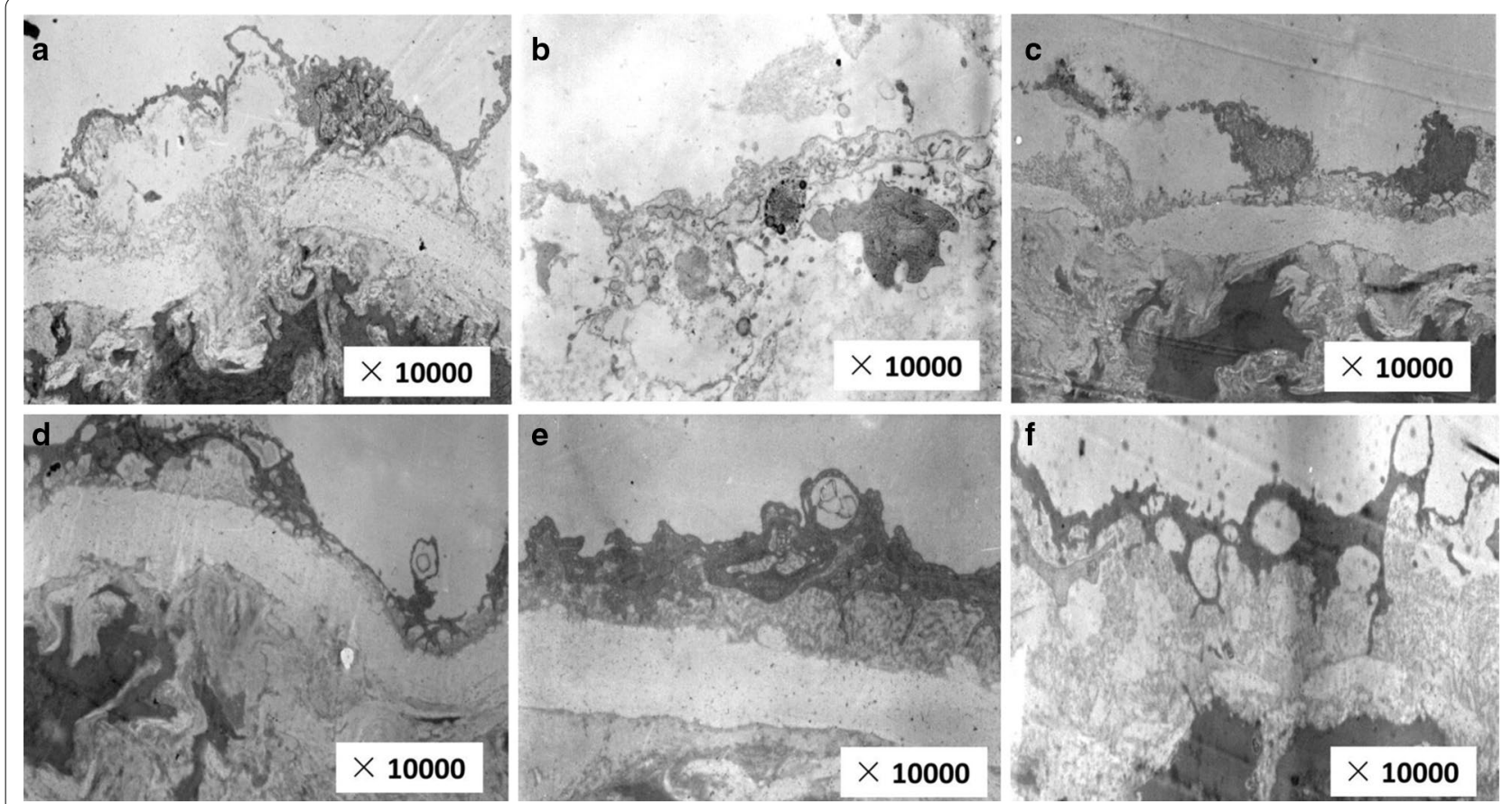

Fig. 6 Representative pictures of electron micrographs $(10,000 \times)$ of cardiac microvascular from the rats of each group. a Normal group, b DCM model group, $\mathbf{c} 8$ cycles group, $\mathbf{d} 18$ cycle group, e 26 cycle group, $\mathbf{f} 36$ cycle group

Table 1 Basic Echocardiographic data of the rats in each group $(\bar{x} \pm s)$

\begin{tabular}{lcccccc}
\hline Characteristics & Normal & DCM model & \multicolumn{2}{l}{ US + MB Subgroups } & & \\
\cline { 5 - 7 } & & & $\mathbf{8}$ cycle & 18 cycle & 26 cycle & 36 cycle \\
\hline LVIDd (mm) & $7.42 \pm 0.21$ & $7.44 \pm 0.41$ & $7.36 \pm 0.48$ & $7.23 \pm 0.69$ & $7.41 \pm 0.78$ & $7.61 \pm 0.69$ \\
IVS (mm) & $1.61 \pm 0.16$ & $1.54 \pm 0.19$ & $1.51 \pm 0.14$ & $1.62 \pm 0.17$ & $1.55 \pm 0.18$ & $1.59 \pm 0.18$ \\
LVEF (\%) & $73.51 \pm 4.44 \#$ & $57.76 \pm 6.03^{*}$ & $64.48 \pm 5.12^{* \#}$ & $65.59 \pm 6.27^{* \#}$ & $68.97 \pm 4.84^{\#}$ & $63.49 \pm 5.34^{* \#}$ \\
LVFS (\%) & $35.06 \pm 3.15 \#$ & $27.70 \pm 3.24^{*}$ & $29.94 \pm 3.55^{* \#}$ & $31.88 \pm 4.28^{* \#}$ & $34.01 \pm 3.97^{\#}$ & $30.78 \pm 3.87^{* \#}$ \\
HR & $378.30 \pm 14.44^{\#}$ & $288.78 \pm 15.82^{*}$ & $301.67 \pm 16.68^{*}$ & $321.21 \pm 29.58^{* \#}$ & $331.13 \pm 20.95^{* \#}$ & $307.42 \pm 23.81^{* \#}$ \\
\hline
\end{tabular}

LVIDd, left ventricular internal dimensions; IVS, interventricular septum dimensions; LVEF, left ventricular ejection fraction; LVFS, percentage of left ventricular fractional shortening; $\mathrm{HR}$, heart rate

${ }^{*} P<0.05$, VS normal group; $\# P<0.05$, VS model group

difference in IVS and LVIDd in UTMD group and DCM model group with a $P$ value $>0.05$ after the intervention.

\section{Myocardial capillary density}

As shown in Finger 7, the DCM group demonstrated a gradual decrease in the MCD of myocardial tissue when compared with that in the control group $(P<0.05)$. Moreover, each US-MBs intervention group showed significant increase in MCD than that in the DCM control group, but the increase of MCD in 8 cycle group had no statistical significance when compared to the DCM control group $(P>0.05)$, while the other US-MB groups were significantly higher than that in the DCM model group
$(P<0.05)$. Among all the US-MB groups, the level of MCD in the 26 cycle group showed no statistically significant difference compared to the normal group $(P>0.05)$.

\section{Discussion}

Microcirculatory dysfunction is believed to play an important role in DCM. Accumulating evidence has demonstrated a progressive reduction of the microvasculature and an impaired angiogenic response to chronic ischemia with the development of diabetes [8]. Recently, low-intensity ultrasound in combination with microbubbles has been shown to stimulate endogenous vascular growth factor, inducing angiogenesis, used in ischemic 
diseases [5, 21, 22]. As gas-filled colloidal materials, SnonVue MBs consisted of an inert gas core and shell composition of phospholipid, polymer with a typical mean diameter of $2.5 \mu \mathrm{m}$. It is a biocompatible and biodegradable material that has been extensively utilized in clinics with sufficient bio-safety. In this study, we evaluated the in vivo therapeutic effects of SonoVue /UTMD combined treatment in a rat model of DCM and explored optimal parameters of ultrasound devices in different group settings.

In this study, both the blood glucose levels (Fig. 3) and histological changes of the pancreas and hearts (Figs. 4, 5) proved that the DCM rats were successfully induced after modeling. Then the rats were divided into six groups according to the different interventions. After all treatments, as shown in Table 1, a significant decrease of the LVEF, LVFS and HR were observed in the DM model group compared with the normal control group $(P<0.05)$. Consistent with previous report [13, 23], the diabetic rats left untreated for 12 weeks were characterized by a declined systolic myocardial performance and had a set of structural abnormalities in the heart compared to the control group. Therefore, it is very important to prevent the occurrence of DCM by intervening before the obvious pathological changes in myocardium in DCM rats.

All of the echocardiography evaluation criteria (Table 1) showed that the levels of LVEF and LVFS were significantly improved, along with a good change of HR between the US-MBs treatment group and the DM model group, while LVEF, LVFS and HR in the 26 cycle group were significantly higher than those in the other US-MB intervention groups. The results indicated that UTMD combined with Sonovue MBs could significantly restore the cardiac functions in DCM animals, especially when the US pulse length parameter is 26 cycles. The histochemical staining data presented in Figs. 5, 6 and 7 showed signs of moderate structural recovery in the US $+\mathrm{MB}$ treatment group compared to the DCM model group, further suggested that the improved functions were the results of structural remodeling of the cardiac tissues and thus may bring longer lasting therapeutic benefit.

US-MBs intervention could increase microvascular density, reverse the ultrastructures of myocardial microvascular injury, improve reparative neovascularization and increase cardiac perfusion. As the diabetes disease progresses, the microangiopathy exerts changes in the morphology and density of microvasculature [24]. As shown in Fig. 6, alterations in the surface of cardiac microvascular and destruction in endothelial cell membrane were observed in the myocardial samples by electron microscopy in DCM rats compared with the normal control ones. There were similar alterations in the ultrastructures of the surface of cardiac microvascular, as observed by electron microscope, in the group with UT-MBs treatment compared with the DCM group (Fig. 6). In our study, the CD 31 immunohistochemical assay (Fig. 7) also confirmed comparable therapeutic effect against DCM-related microangiopathy. The complex mechanism of angiogenesis using USMBs treated in DCM model remains to be explored, it may be related to two factors. First of all, cavitation and mechanical effects of blasting could increase oxidative stress and induce local inflammatory factors, and then release vascular growth factors to activate angiogenesis [21]. Secondly, increasing the blood flow using the USMBs treatment is most likely mediated by cavitationrelated increases in shear and activation of endothelial nitric oxide synthase [25]. Huang et al. [26] suggested that the PI3K/Akt signal pathway might participate in modulating the activity of eNOS. Akt signaling protects against myocyte apoptosis induced by cardiac ischemia-reperfusion injury and DCM [27, 28]. The protective mechanism of US-MBs promoting angiogenesis may be associated with the activation of the PI3K/ Akt signaling pathway by the up-regulation of expression of phosphorylated Akt protein to activate eNOS [27, 29].

Among various US-MBs treated groups, we found that different pulse length could achieve different intervention results. There were well-integrated ultrastructures in the myocardial samples in the 26 cycle group compared with other US-MBs treatment and DCM group, while it also demonstrated the highest effectiveness in increasing density of microvasculature. Furthermore, the routine echocardiography (Table 1) showed that the 26 cycle group significantly restored the cardiac functions in DCM rats. Therefore, this method can be used as an effective strategy to prevent the deterioration of cardiac function in DCM rats. This pre-clinical study provides us with great hope for early intervention of DCM in diabetic patients in the future. We predict that once the delivery system is optimized, this combined strategy might be used as an effective option for preventing DCM development.

\section{Conclusion}

In conclusion, while using non-viral vectors SnonVue MBs combined with UTMD technique can significantly improve or even reverse cardiac dysfunction and pathological abnormalities, especially using the 26 cycle parameters. Under further study, this combined strategy might be a promising option for early intervention of DCM in diabetic patients. 

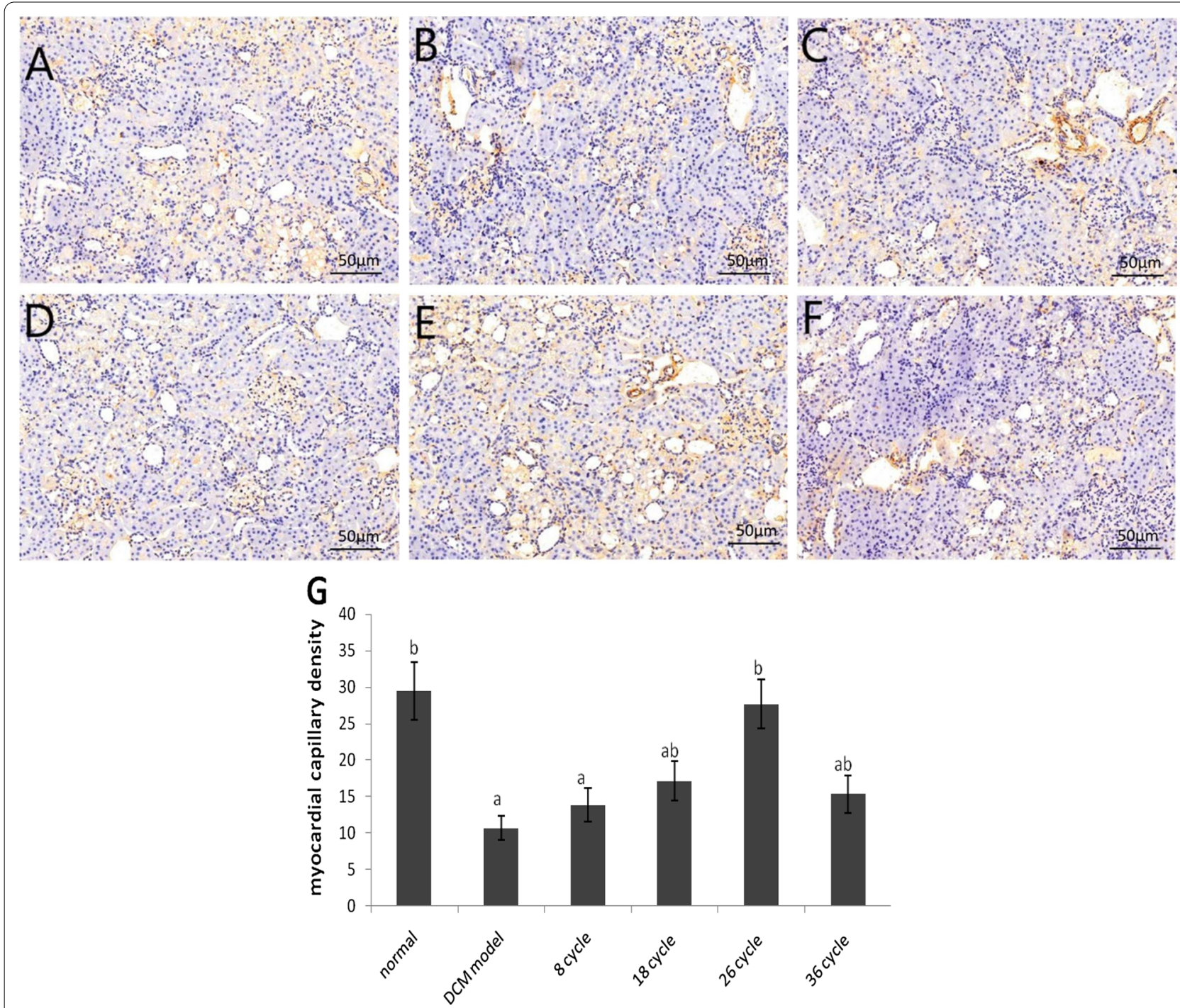

Fig. 7 Representatives of CD31 immunohistochemical staining of MCD and the semi-quantitative analysis for all groups (400x). A normal group, B DCM model group, C 8 cycles group, D 18 cycle group, E 26 cycle group, $\mathbf{F} 36$ cycle group, $\mathbf{G}$ Statistical histogram. a $P<0.05$ versus the normal control group, $\mathbf{b} P<0.05$ versus the DM model group

\section{Abbreviations}

DM: Diabetes mellitus; DCM: Diabetic cardiomyopathy; UTMD: Ultrasoundtargeted microbubble destruction; bFGF: Basic fibroblast growth factor; MBs: Microbubbles; MB: Microbubble; STZ: Streptozotocin; LVEF: Left ventricular ejection fraction; LVIDd: Left ventricular end-diastolic diameter; IVS: Interventricular septum dimensions; LVFS: Left ventricular fraction shortening; HE: Hematoxylin and eosin; MCD: Myocardial capillary density; HR: Heart rate.

\section{Acknowledgements}

The authors would like to thank the Central Laboratory of Henan Provincial People's Hospital for their technical assistance.

\section{Author's contributions}

$X Z$ and $X T$ contributed equally in writing the manuscript and share first authorship. XZ, NZ and YY have participated in the ultrasound treatment and data anslysis. PL and $\mathrm{HZ}$ corrected and organized the collected data. YJ and ZF collected of the samples, made animal model and histology examination. $J Y$ and XT designed of this study and revised the manuscript. All authors read and approved the final manuscript.

\section{Funding}

This work was supported by the National Natural Science Foundation of China (Grant Nos. U1704175, 81571696) from Xinqiao Tian, Health and Family Planning Commission of Henan Province (Grant No. SB201901093) from Haohui Zhu, Science and Technology New Star Project of Chinese Ultrasound Doctors Association (Grant No.KJXX2019006) from Xijun Zhang. The funding sources helps in the design of the study, data collection, analysis and report writing. 


\section{Availability of data and material}

The datasets used or analysed during the current study are available from the corresponding author on reasonable request.

\section{Ethics approval and consent to participate}

All study protocol and image procedures have been approved by the Anima Care and Use Committee of Xinxiang Medical College.

\section{Consent for publication}

Not applicable.

\section{Competing interests}

The authors have declared that no competing interest exists.

\section{Author details}

${ }^{1}$ Department of Ultrasonography, Zhengzhou University People's Hospital, Henan Provincial People's Hospital, Henan University People's Hospital, NO. 7 Weiwu Road, Zhengzhou 450003, Henan, China. ${ }^{2}$ School of Pharmacy, Xinxiang Medical University, Xinxiang 453002, Henan, China.

Received: 2 September 2020 Accepted: 8 December 2020

Published online: 07 January 2021

\section{References}

1. Mazzone T, Chait A, Plutzky J. Cardiovascular disease risk in type 2 diabetes mellitus: insights from mechanistic studies. Lancet (London, England). 2008:371:1800-9.

2. Evangelista I, Nuti R, Picchioni T, Dotta F, Palazzuoli A. Molecular dysfunction and phenotypic derangement in diabetic cardiomyopathy. Int J Mol Sci. 2019;20:3264

3. Gaurav SG, Athithan L, Gerry PM. Diabetic cardiomyopathy: prevalence, determinants and potential treatments. Ther Adv Endocrinol Metab. 2019:10:2042018819834869.

4. Boodhwani M, Neel RS, Mieno S, Xu SH, Feng J, Ramlawi B, et al. Functional, cellular, and molecular characterization of the angiogenic response to chronic myocardial ischemia in diabetes. Circulation. 2007;116:131-7.

5. Zheng L, Shen CL, Li JM, Ma YL, Yan N, Tian XQ, et al. Assessment of the preventive effect against diabetic cardiomyopathy of FGF1-loaded nanoliposomes combined with microbubble cavitation by ultrasound. Front Pharmacol. 2019;10:1535.

6. Sara N, Edna S, João F, Sofia V, Eugénia C, Frederico CP, et al. Early cardiac changes in a rat model of prediabetes: brain natriuretic peptide overexpression seems to be the best marker. Cardiovasc Diabetol. 2013;12:44-44.

7. Sypalo A, Kravchun P, Kadykova O. The influence of mono- and multivascular lesions of coronary arteries on the course of coronary heart disease in patients with diabetes mellitus type 2. Georgian Med News. 2017:264:61-5.

8. Hinkel R, Howe A, Renner S, Ng J, Lee S, Klett K, et al. Diabetes mellitusinduced microvascular destabilization in the myocardium. J Am Coll Cardiol. 2017;69:131-43

9. Singla D, Wang J. Fibroblast growth factor-9 activates c-Kit progenitor cells and enhances angiogenesis in the infarcted diabetic heart. Oxidative Med Cell Longev. 2016;2016:5810908.

10. Dai XZ, Yan XQ, Zeng J, Chen J, Wang YH, Chen J, et al. Elevating CXCR7 improves angiogenic function of EPCs via Akt/GSK-3ß/Fyn-Mediated Nrf2 activation in diabetic limb ischemia. Circ Res. 2017;120:e7-23.

11. Teng $X$, Ji C, Zhong H, Zheng D, Ni R, Hill DJ, et al. Selective deletion of endothelial cell calpain in mice reduces diabetic cardiomyopathy by improving angiogenesis. Diabetologia. 2019;62:860-72.

12. Zhao $Y Z$, Tian XQ, Zhang M, Cai L, Ru A, Shen XT, et al. Functional and pathological improvements of the hearts in diabetes model by the combined therapy of bFGF-loaded nanoparticles with ultrasound-targeted microbubble destruction. J Controll Release Off J Controll Release Soc. 2014;186:22-31.
13. Zhao $Y Z$, Zhang M, Ho LW, Tian XQ, Zheng L, Yu XC, et al. Prevent diabetic cardiomyopathy in diabetic rats by combined therapy of aFGF-loaded nanoparticles and ultrasound-targeted microbubble destruction technique. J Controll Release Off J Controll Release Soc. 2016;223:11-21.

14. Dietrich CF. Comments and illustrations regarding the guidelines and good clinical practice recommendations for contrast-enhanced ultrasound (CEUS) —update 2008. Ultraschall Med. 2008;29:S188-202.

15. Dietrich CF, Nolsøe CP, Barr RG, Berzigotti A, Burns PN, Cantisani V, et al. Guidelines and good clinical practice recommendations for contrast enhanced ultrasound (CEUS) in the liver-update 2020-WFUMB in cooperation with EFSUMB, AFSUMB, AIUM, and FLAUS. Ultraschall Med. 2020:41:562-85.

16. Thomsen HS. European Society of Urogenital Radiology (ESUR) guidelines on the safe use of iodinated contrast media. Eur J Radiol. 2006:60:307-13.

17. Wang XY, Pan JY, Liu H, Zhang MJ, Liu D, Lu L, et al. AIM2 gene silencing attenuates diabetic cardiomyopathy in type 2 diabetic rat model. Life Sci. 2019;221:249-58

18. Xue JT, Shi YL, Li CY, Xu XJ, Xu SX, Cao MX. Methylcellulose and polyacrylate binary hydrogels used as rectal suppository to prevent type I diabetes. Colloids Surf B Biointerfaces. 2018;172:37-42.

19. Zheng WC, Li D, Gao X, Zhang WQ, Barry OR. Carvedilol alleviates diabetic cardiomyopathy in diabetic rats. Exp Therap Med. 2019;17:479-87.

20. Huang R, Shi ZD, Chen L, Zhang YQ, Li J, An Y. Rutin alleviates diabetic cardiomyopathy and improves cardiac function in diabetic ApoEknockout mice. Eur J Pharmacol. 2017:814:151-60.

21. Hanawa K, Ito K, Aizawa K, Shindo T, Nishimiya K, Hasebe Y, et al. Lowintensity pulsed ultrasound induces angiogenesis and ameliorates left ventricular dysfunction in a porcine model of chronic myocardial ischemia. PLOS ONE. 2014:9:e104863.

22. Xue JT, Zhou NQ, Yang YP, Jing Y, Qiu Y, Liu YH, et al. Puerarin-loaded ultrasound microbubble contrast agent used as sonodynamic therapy for diabetic cardiomyopathy rats. Colloids Surf B Biointerfaces. 2020;190:110887.

23. Tian XQ, Ni XW, Xu HL, Zheng L, ZhuGe DL, Chen B, et al. Prevention of doxorubicin-induced cardiomyopathy using targeted MaFGF mediated by nanoparticles combined with ultrasound-targeted MB destruction. Int J Nanomed. 2017:12:7103-19.

24. Kawaguchi M, Techigawara M, Ishihata T, Asakura T, Saito F, Maehara K, et al. A comparison of ultrastructural changes on endomyocardial biopsy specimens obtained from patients with diabetes mellitus with and without hypertension. Heart Vessels. 1997:12:267-74.

25. Todd BJ, Brian HM, Xie A, Zhao Y, Kim S, Nathan JL, et al. Augmentation of limb perfusion and reversal of tissue ischemia produced by ultrasound-mediated microbubble cavitation. Circ Cardiovasc Imaging. 2015;8:e002979.

26. Huang JJ, Shi YQ, Li RL, Hu A, Lu ZY, Weng L, et al. Angiogenesis effect of therapeutic ultrasound on HUVECs through activation of the PI3K-AkteNOS signal pathway. Am J Transl Res. 2015;7:1106-15.

27. Zhang M, Yu WZ, Shen XT, Xiang Q, Xu J, Yang JJ, et al. Advanced interfere treatment of diabetic cardiomyopathy rats by aFGF-loaded heparinmodified microbubbles and UTMD technique. Cardiovasc Drugs Ther 2016:30:247-61.

28. Chen YP, Ba LN, Huang W, Liu Y, Pan H, Mingyao E, et al. Role of carvacrol in cardioprotection against myocardial ischemia/reperfusion injury in rats through activation of MAPK/ERK and Akt/eNOS signaling pathways. Eur J Pharmacol. 2017:796:90-100.

29. Xu H, Xu H, Hai GF, Qing HE, Zhang C. Protective effect of modified human acidic fibroblast growth factor against actinomycin D-induced NRK52E cells apoptotic death. Trop J Pharm Res. 2013;12:343-9.

\section{Publisher's Note}

Springer Nature remains neutral with regard to jurisdictional claims in published maps and institutional affiliations. 